\title{
Existence and Uniqueness of Solutions of a Nonlinear Heat Equation
}

M.A. RINCON ${ }^{1}$, Instituto de Matemática, Universidade Federal do Rio de Janeiro, Rio de Janeiro, RJ, Brazil

J. LÍMACO ${ }^{2}$, Instituto de Matemática, Universidade Federal Fluminense, Niteroi, RJ, Brazil

I-SHIH LIU 3 , Instituto de Matemática, Universidade Federal do Rio de Janeiro, Rio de Janeiro, RJ, Brazil.

\begin{abstract}
A nonlinear partial differential equation of the following form is considered:

$$
u^{\prime}-\operatorname{div}(a(u) \nabla u)+b(u)|\nabla u|^{2}=0
$$

which arises from the heat conduction problems with strong temperature-dependent material parameters, such as mass density, specific heat and heat conductivity. Existence, uniqueness and asymptotic behavior of initial boundary value problems under appropriate assumptions on the material parameters are established.
\end{abstract}

\section{Introduction}

Metallic materials present a complex behavior during heat treatment processes involving phase changes. In a certain temperature range, change of temperature induces a phase transformation of metallic structure, which alters physical properties of the material. Indeed, measurements of specific heat and conductivity show a strong temperature dependence during processes such as quenching of steel.

In this paper, we are interested in a nonlinear heat equation with temperaturedependent material parameters, in contrast to the usual linear heat equation with constant coefficients.

Let $\theta(x, t)$ be the temperature field, then we can write the conservation of energy in the following form:

$$
\rho \varepsilon^{\prime}+\operatorname{div} q=0,
$$

where prime denotes the time derivative.

\footnotetext{
${ }^{1}$ rincon@dcc.ufrj.br

2juanbrj@hotmail.com.br

${ }^{3}$ liu@im.ufrj.br
} 
The mass density $\rho=\rho(\theta)>0$ may depend on temperature due to possible change of material structure, while the heat flux $q$ is assumed to be given by the Fourier law with temperature-dependent heat conductivity,

$$
q=-\kappa \nabla \theta, \quad \kappa=\kappa(\theta)>0 .
$$

The internal energy density $\varepsilon=\varepsilon(\theta)$ generally depends on the temperature, and the specific heat $c$ is assumed to be positive defined by $c(\theta)=\partial \varepsilon / \partial \theta>0$, which is not necessarily a constant.

We can reformulate the equation (1.1) in terms of the energy $\varepsilon$ instead of the temperature $\theta$. Rewriting Fourier law as

$$
q=-\kappa(\theta) \nabla \theta=-\alpha(\varepsilon) \nabla \varepsilon,
$$

and observing that $\nabla \varepsilon=\partial \varepsilon / \partial \theta \nabla \theta=c(\theta) \nabla \theta$, we have $c(\theta) \alpha(\varepsilon)=\kappa(\theta)$, and hence $\alpha=\alpha(\varepsilon)>0$.

Now let $u$ be defined as $u=\varepsilon(\theta)$, then the equation (1.1) becomes

$$
\rho(u) u^{\prime}-\operatorname{div}(\alpha(u) \nabla u)=0 .
$$

Since $\rho(u)>0$, dividing the equation by $\rho$, we obtain

$$
u^{\prime}-\operatorname{div}\left(\frac{\alpha(u) \nabla u}{\rho(u)}\right)+\left(\nabla \frac{1}{\rho(u)}\right) \cdot(\alpha(u) \nabla u)=0 .
$$

Since $c=\frac{\partial \varepsilon}{\partial \theta}=\frac{d u}{d \theta}$

$$
\left(\nabla \frac{1}{\rho(u)}\right)=-\frac{1}{\rho(u)^{2}} \frac{d \rho(u)}{d u} \nabla u=-\frac{1}{\rho(u)^{2}} \frac{d \rho}{d \theta} \frac{d \theta}{d u} \nabla u=-\frac{1}{\rho(u)^{2}} \frac{d \rho}{d \theta} \frac{1}{c} \nabla u .
$$

Substituting into equation (1.4) we obtain

$$
u^{\prime}-\operatorname{div}\left(\frac{\alpha(u) \nabla u}{\rho(u)}\right)-\left(\frac{1}{\rho(u)^{2}} \frac{d \rho}{d \theta} \frac{1}{c} \nabla u\right) \cdot(\alpha(u) \nabla u)=0
$$

which is equivalent to

$$
u^{\prime}-\operatorname{div}(a(u) \nabla u)+b(u)|\nabla u|^{2}=0,
$$

where $a(u)=\frac{\alpha(u)}{\rho(u)}=\frac{\alpha(u) c(u)}{\rho(u) c(u)}=\frac{k(u)}{\rho(u) c(u)}>0 \quad$ and $\quad b(u)=-\frac{\alpha(u)}{c \rho(u)^{2}} \frac{d \rho}{d \theta}>0$. The positiveness of $a(u)$ and $b(u)$ is the consequence of thermodynamic considerations (see [8]), and reasonable physical experiences: the specific heat $c>0$, the thermal conductivity $\kappa>0$, the mass density $\rho>0$, and the thermal expansion $d \rho / d \theta<0$. In this paper we shall formulate the problem based on the nonlinear heat equation (1.5). 


\subsection{Formulation of the problem}

Let $\Omega$ be a bounded open set of $\mathbb{R}$, with regular boundary. We represent by $Q=$ $\Omega \times(0, T)$ for $T>0$, a cylindrical domain, whose lateral boundary we re'present by $\Sigma=\Gamma \times(0, T)$. We shall consider the following non-linear problem:

$$
\left\{\begin{array}{l}
u^{\prime}-\operatorname{div}(a(u) \nabla u)+b(u)|\nabla u|^{2}=0 \quad \text { in } Q \\
u=0 \quad \text { on } \Sigma, \quad u(x, 0)=u_{0}(x) \quad \text { in } \Omega
\end{array}\right.
$$

Mathematical models of semi-linear and nonlinear parabolic equations under Dirichlet or Neumann boundary conditions has been considered in several papers, among them, let us mention $([1],[2],[3])$ and ([4], [5], [9]), respectively.

Feireisl et al [6] prove that with non-negative initial data, the function $a(u) \equiv 1$ and $g(u, \nabla u) \leq h(u)\left(1+|\nabla u|^{2}\right)$, instead of the non-linear term $b(u)|\nabla u|^{2}$ in (1.6), there exists an admissible solution positive in some interval $\left[0, T_{\max }\right)$ and if $T_{\max }<$ $\infty$ then $\lim _{t \rightarrow T_{\max }}\|u(t, .)\|_{\infty}=\infty$.

For Problem (1.6) we will prove global existence, uniqueness and asymptotic behavior for the one-dimensional case.

\section{Existence and Uniqueness}

Let $((\cdot, \cdot)),\|\cdot\|$ and $(\cdot, \cdot),|\cdot|$ be respectively the scalar product and the norms in $H_{0}^{1}(\Omega)$ and $L^{2}(\Omega)$. Thus, when we write $|u|=|u(t)|,\|u\|=\|u(t)\|$ its means the $L^{2}(\Omega), H_{0}^{1}(\Omega)$ norm of $u(x, t)$ respectively. To prove the existence and uniqueness of solutions for the one-dimensional case, we need the following hypotheses:

H1: $a(u)$ and $b(u)$ belongs to $C^{1}(\mathbb{R})$ and there are positive constants $a_{0}, a_{1}$ such that, $a_{0} \leq a(u) \leq a_{1}$ and $b(u) u \geq 0$.

H2: There is a positive constant $M>0$ such that $\max _{s \in \mathbb{R}}\left\{\left|\frac{d a}{d u}(s)\right| ;\left|\frac{d b}{d u}(s)\right|\right\} \leq M$

H3: $u_{0} \in H_{0}^{1}(\Omega) \cap H^{2}(\Omega)$ such that $\left|\Delta u_{0}\right|<\varepsilon$, for some constant $\varepsilon>0$.

Remark. From hypothesis (H1), we have that $b(u) u \geq 0, \forall u \in \mathbb{R}$. Thus from hypothesis $(\mathrm{H} 2),|b(u)| \leq M|u|$.

Theorem 2.1. Under the hypotheses (H1), (H2) and (H3), there exist a positive constant $\varepsilon_{0}$ such that, if $0<\varepsilon \leq \varepsilon_{0}$ then the problem (1.6) admits a unique solution $u: Q \rightarrow \mathbb{R}$, satisfying the following conditions:

i. $u \in L^{2}\left(0, T ; H_{0}^{1}(\Omega) \cap H^{2}(\Omega)\right), \quad u^{\prime} \in L^{2}\left(0, T ; H_{0}^{1}(\Omega)\right)$

ii. $u^{\prime}-\operatorname{div}(a(u) \nabla u)+b(u)|\nabla u|^{2}=0, \quad$ in $L^{2}(Q)$,

iii. $u(0)=u_{0}$.

Remark. The positive constant $\varepsilon_{0}$ will be determined later (see (2.14), (2.15), (2.20) and (3.11)). 
Proof. To prove the theorem, we employ Galerkin method with the Hilbertian basis from $H_{0}^{1}(\Omega)$, given by the eigenvectors $\left(w_{j}\right)$ of the spectral problem: $\left(\left(w_{j}, v\right)\right)=$ $\lambda_{j}\left(w_{j}, v\right)$ for all $v \in V$ and $j=1,2, \cdots$. We represent by $V_{m}$ the subspace of $V$ generated by vectors $\left\{w_{1}, w_{2}, \ldots, w_{m}\right\}$. We propose the following approximate problem: Determine $u_{m} \in V_{m}$, so that

$$
\left\{\begin{array}{l}
\left(u_{m}^{\prime}, v\right)+\left(a\left(u_{m}\right) \nabla u_{m}, \nabla v\right)+\left(b\left(u_{m}\right)\left|\nabla u_{m}\right|^{2}, v\right)=0 \quad \forall v \in V_{m}, \\
u_{m}(0)=u_{0 m} \rightarrow u_{0} \quad \text { in } \quad H_{0}^{1}(\Omega) \cap H^{2}(\Omega) .
\end{array}\right.
$$

\section{Existence}

The system of ordinary differential equations (2.1) has a local solution in the interval $\left(0, T_{m}\right)$. To extend the local solution to the interval $(0, T)$ independent of $m$ the following a priori estimates are needed.

Estimate I: Taking $v=u_{m}(t)$ in the equation (2.1) and integrating over $(0, T)$, we obtain

$$
\frac{1}{2}\left|u_{m}\right|^{2}+a_{0} \int_{0}^{T}\left\|u_{m}\right\|^{2}+\int_{0}^{T} \int_{\Omega} b\left(u_{m}\right) u_{m}\left|\nabla u_{m}\right|^{2}<\frac{1}{2}\left|u_{0}\right|^{2}
$$

where we have used hypothesis (H1). Taking $\hat{a}_{0}=\min \left\{a_{0}, \frac{1}{2}\right\}>0$, we obtain

$$
\left|u_{m}\right|^{2}+\int_{0}^{T}\left\|u_{m}\right\|^{2}+\int_{0}^{T} \int_{\Omega} b\left(u_{m}\right) u_{m}\left|\nabla u_{m}\right|^{2}<\frac{1}{2 \hat{a}_{0}}\left|u_{0}\right|^{2} .
$$

Thus, applying the Gronwall's inequality in (2.3) yields

$$
\left(u_{m}\right) \quad \text { is bounded in } \quad L^{\infty}\left(0, T ; L^{2}(\Omega)\right) \cap L^{2}\left(0, T ; H_{0}^{1}(\Omega)\right) .
$$

Estimate II: Taking $v=u_{m}^{\prime}$ in the equation $(2.1)_{1}$ and integrating over $(0, T)$, we obtain

$$
2\left|u_{m}^{\prime}\right|^{2}+\frac{d}{d t} \int_{\Omega} a\left(u_{m}\right)\left|\nabla u_{m}\right|^{2}=\int_{\Omega} \frac{d a}{d u}\left(u_{m}\right) u_{m}^{\prime}\left|\nabla u_{m}\right|^{2}+2 \int_{\Omega} b\left(u_{m}\right) u_{m}^{\prime}\left|\nabla u_{m}\right|^{2} .
$$

On the other hand, from hypothesis (H2), we have the following inequality,

$$
\frac{1}{2} \int_{\Omega} \frac{d a}{d u}\left(u_{m}\right) u_{m}^{\prime}\left|\nabla u_{m}\right|^{2} \leq \frac{1}{2} M C_{0}\left\|u_{m}^{\prime}\right\|\left\|u_{m}\right\|^{2},
$$

and since $|\cdot|_{L^{\infty}(\Omega)} \leq C_{0}\|\cdot\|$ and $\left|b\left(u_{m}\right)\right| \leq M\left|u_{m}\right|$, we obtain

$$
\int_{\Omega} b\left(u_{m}\right) u_{m}^{\prime}\left|\nabla u_{m}\right|^{2} \leq M C_{0}^{2}\left\|u_{m}^{\prime}\right\|\left\|u_{m}\right\|^{3}
$$

where $C_{0}=C_{0}(\Omega)$ is a constant depending on $\Omega$. 
Substituting (2.6) and (2.7) into the left hand side of (2.5) we get

$$
\begin{aligned}
\left|u_{m}^{\prime}\right|^{2} & +\frac{1}{2} \frac{d}{d t} \int_{\Omega} a\left(u_{m}\right)\left|\nabla u_{m}\right|^{2} \leq \frac{1}{2} M C_{0}\left\|u_{m}^{\prime}\right\|\left\|u_{m}\right\|^{2}+M C_{0}^{2}\left\|u_{m}^{\prime}\right\|\left\|u_{m}\right\|^{3} \\
& \leq \frac{1}{4}\left(M C_{0}\right)^{2}\left\|u_{m}^{\prime}\right\|^{2}\left\|u_{m}\right\|^{2}+\frac{1}{2}\left\|u_{m}\right\|^{2}+\left(M C_{0}^{2}\right)^{2}\left\|u_{m}^{\prime}\right\|^{2}\left\|u_{m}\right\|^{4} .
\end{aligned}
$$

Now taking the derivative of the equation $(2.1)_{1}$ with respect to $t$ and making $v=u_{m}^{\prime}$, we have

$$
\begin{aligned}
& \frac{1}{2} \frac{d}{d t}\left|u_{m}^{\prime}\right|^{2}+\int_{\Omega} a\left(u_{m}\right)\left|\nabla u_{m}^{\prime}\right|^{2}=-\int_{\Omega} \frac{d b}{d u}\left(u_{m}\right)\left|u_{m}^{\prime}\right|^{2}\left|\nabla u_{m}\right|^{2}-\int_{\Omega} \frac{d a}{d u}\left(u_{m}\right) u_{m}^{\prime}\left|\nabla u_{m}\right|^{2} \\
& -2 \int_{\Omega} b\left(u_{m}\right) \nabla u_{m} \nabla u_{m}^{\prime} u_{m}^{\prime} \leq M C_{0}^{2}(3+M)\left\|u_{m}^{\prime}\right\|^{2}\left\|u_{m}\right\|^{2}+\frac{1}{2}\left\|u_{m}\right\|^{2} .
\end{aligned}
$$

From inequalities (2.8) and (2.9), we have

$$
\begin{gathered}
\frac{d}{d t}\left\{\frac{1}{2}\left|u_{m}^{\prime}\right|^{2}+\frac{1}{2} \int_{\Omega} a\left(u_{m}\right)\left|\nabla u_{m}\right|^{2}\right\}+\left|u_{m}^{\prime}\right|+\frac{a_{0}}{2}\left\|u_{m}^{\prime}\right\|^{2}+ \\
\left\|u_{m}^{\prime}\right\|^{2}\left\{\frac{a_{0}}{2}-\alpha_{0}\left\|u_{m}\right\|^{4}-\alpha_{1}\left\|u_{m}\right\|^{2}\right\} \leq\left\|u_{m}\right\|^{2},
\end{gathered}
$$

where we have defined $\alpha_{0}=\left(M C_{0}^{2}\right)^{2}$ and $\alpha_{1}=M C_{0}\left(\frac{1}{4} M C_{0}+3 C_{0}+1\right)$.

Now, under the condition that the following inequality,

$$
\alpha_{0}\left\|u_{m}\right\|^{4}+\alpha_{1}\left\|u_{m}\right\|^{2}<\frac{a_{0}}{4} \quad \forall t \geq 0,
$$

be valid, the coefficients of the term $\left\|u_{m}^{\prime}\right\|$ in the relation (2.10) is positive and we can integrate it with respect to $t$,

$$
\left|u_{m}^{\prime}\right|^{2}+\int_{\Omega} a\left(u_{m}\right)\left|\nabla u_{m}\right|^{2}+\int_{0}^{t}\left|u_{m}^{\prime}\right|^{2}+a_{0} \int_{0}^{t}\left\|u_{m}^{\prime}\right\|^{2} \leq C .
$$

Therefore, applying the Gronwall's inequality in (2.12), we obtain the following estimate:

$$
\left(u_{m}^{\prime}\right) \quad \text { is bounded in } \quad L^{\infty}\left(0, T ; L^{2}(\Omega)\right) \cap L^{2}\left(0, T ; H_{0}^{1}(\Omega)\right) .
$$

Now we want to prove that the inequality (2.11) is valid if the initial data are sufficiently small. Suppose that

$$
\frac{\alpha_{0}}{a_{0}^{2}}\left\{S_{0}+a_{1}\left\|u_{0}\right\|^{2}+\frac{1}{a_{0}}\left|u_{0}\right|^{2}\right\}^{2}+\frac{\alpha_{1}}{a_{0}}\left\{S_{0}+a_{1}\left\|u_{0}\right\|^{2}+\left\|u_{0}\right\|^{2}\right\}<\frac{a_{0}}{4}
$$

and

$$
\alpha_{0}\left\|u_{0}\right\|^{4}+\alpha_{1}\left\|u_{0}\right\|^{2}<\frac{a_{0}}{4}
$$

where we have denoted $S_{0}=\left(M\left(\left\|u_{0}\right\|+\left|\Delta u_{0}\right|^{2}+\left\|u_{0}\right\|\left|\Delta u_{0}\right|^{2}\right)+a_{1}\left|\Delta u_{0}\right|\right)^{2}$. 
We shall prove by contradiction. Suppose that (2.11) is false, then there is a $t^{*}$ such that

$$
\alpha_{0}\left\|u_{m}(t)\right\|^{4}+\alpha_{1}\left\|u_{m}(t)\right\|^{2}<\frac{a_{0}}{4} \quad \text { if } \quad 0<t<t^{*}
$$

and

$$
\alpha_{0}\left\|u_{m}\left(t^{*}\right)\right\|^{4}+\alpha_{1}\left\|u_{m}\left(t^{*}\right)\right\|^{2}=\frac{a_{0}}{4} .
$$

Integrating (2.10) from 0 to $t^{*}$, we obtain

$$
\begin{aligned}
\frac{1}{2}\left|u_{m}^{\prime}\left(t^{*}\right)\right|^{2} & +\int_{\Omega} a\left(u_{m}\right)\left|\nabla u_{m}\left(t^{*}\right)\right|^{2} \leq \int_{\Omega} a\left(u_{m}(0)\right)\left|\nabla u_{m}(0)\right|^{2} \\
& +\frac{1}{2}\left|u_{m}^{\prime}(0)\right|^{2}+\int_{0}^{t^{*}}\left\|u_{m}\right\|^{2} \leq a_{1}\left\|u_{0}\right\|^{2}+\frac{1}{a_{0}}\left|u_{0}\right|^{2} \\
& +\left(M\left(\left\|u_{0}\right\|+\left|\Delta u_{0}\right|^{2}+\left\|u_{0}\right\|\left|\Delta u_{0}\right|^{2}\right)+a_{1}\left|\Delta u_{0}\right|^{2}\right)^{2}
\end{aligned}
$$

and consequently, $\quad\left\|u_{m}\left(t^{*}\right)\right\|^{2} \leq \frac{1}{a_{0}} S_{0}+\frac{a_{1}}{a_{0}}\left\|u_{0}\right\|^{2}+\frac{1}{a_{0}^{2}}\left|u_{0}\right|^{2}$. Using (2.14) and (2.15) we obtain

$$
\begin{aligned}
\alpha_{0}\left\|u_{m}\left(t^{*}\right)\right\|^{4} & +\alpha_{1}\left\|u_{m}\left(t^{*}\right)\right\|^{2} \leq \frac{\alpha_{0}}{a_{0}^{2}}\left\{S_{0}+a_{1}\left\|u_{0}\right\|^{2}+\frac{1}{a_{0}}\left|u_{0}\right|^{2}\right\}^{2} \\
& +\alpha_{1}\left\{\frac{1}{a_{0}} S_{0}+\frac{a_{1}}{a_{0}}\left\|u_{0}\right\|^{2}+\frac{1}{a_{0}}\left\|u_{0}\right\|^{2}\right\}<\frac{a_{0}}{4}
\end{aligned}
$$

hence, comparing with (2.17), we have a contradiction.

Estimate III: Taking $v=-\Delta u_{m}(t)$ in the equation $(2.1)_{1}$, and using hypothesis H1, we obtain

$$
\frac{d}{d t}\left\|u_{m}\right\|^{2}+\int_{\Omega} a\left(u_{m}\right)\left|\Delta u_{m}\right|^{2} \leq a_{1}\left\|u_{m}\right\|\left|\Delta u_{m}\right|+b_{1}\left|\Delta u_{m}\right|^{2}\left\|u_{m}\right\|,
$$

where we have used the following inequality,

$$
\begin{aligned}
\int_{\Omega} b\left(u_{m}\right)\left|\nabla u_{m}\right|^{2}\left|\Delta u_{m}\right| & \leq \bar{b}_{1}\left|\nabla u_{m}\right|_{L^{\infty}} \int_{\Omega}\left|\nabla u_{m}\right|\left|\Delta u_{m}\right| \leq \bar{b}_{1}\left|\nabla u_{m}\right|_{H^{1}(\Omega)}\left\|u_{m}\right\|\left|\Delta u_{m}\right| \\
& \leq \bar{b}_{1}\left|\Delta u_{m}\right|\left\|u_{m}\right\|\left|\Delta u_{m}\right|=\bar{b}_{1}\left\|u_{m}\right\|\left|\Delta u_{m}\right|^{2} .
\end{aligned}
$$

In this expression we have denoted $\bar{b}_{1}=\sup |b(s)|$, for all $s \in\left[-\frac{a_{0}}{4 \alpha_{1}}, \frac{a_{0}}{4 \alpha_{1}}\right]$. Note that, from (2.11), we have $\left|u_{m}(t)\right| \leq\left\|u_{m}(t)\right\|<a_{0} / 4 \alpha_{1}$ and $b_{1}=\bar{b}_{1}+M$.

Using hypothesis (H1), we obtain

$$
\frac{d}{d t}\left\|u_{m}\right\|^{2}+\frac{a_{0}}{2}\left|\Delta u_{m}\right|^{2} \leq a_{1}\left\|u_{m}\right\|\left|\Delta u_{m}\right|+b_{1}\left|\Delta u_{m}\right|^{2}\left\|u_{m}\right\|,
$$

or equivalently, $\quad \frac{d}{d t}\left\|u_{m}\right\|^{2}+\left(\frac{a_{0}}{2}-b_{1}\left\|u_{m}\right\|\right)\left|\Delta u_{m}\right|^{2} \leq a_{1}\left\|u_{m}\right\|\left|\Delta u_{m}\right|$. 
Note that the constant $C$ from (2.12) is given by $C=S_{0}+a_{1}\left\|u_{0}\right\|^{2}+\frac{1}{2 a_{0}}\left|u_{0}\right|^{2}$. Considering

$$
b_{1}\left(\frac{1}{a_{0}}\left(S_{0}+a_{1}\left\|u_{0}\right\|^{2}+\frac{1}{2 a_{0}}\left|u_{0}\right|^{2}\right)^{1 / 2}\right)<\frac{a_{0}}{4}
$$

and from (2.12) we obtain $\frac{a_{0}}{4} \leq\left(\frac{a_{0}}{2}-b_{1}\left\|u_{m}\right\|\right)$, it implies that

$$
\frac{d}{d t}\left\|u_{m}\right\|^{2}+\frac{a_{0}}{4}\left|\Delta u_{m}\right|^{2} \leq a_{1}\left\|u_{m}\right\|\left|\Delta u_{m}\right| \leq \frac{a_{0}}{8}\left|\Delta u_{m}\right|^{2}+C\left\|u_{m}\right\|^{2} .
$$

Now, integrating from 0 to $t$, we obtain the estimate: $\left\|u_{m}\right\|^{2}+\int_{0}^{T}\left|\Delta u_{m}\right|^{2} \leq \widehat{C}$. Hence, we have

$$
\left(u_{m}\right) \quad \text { is bounded in } \quad L^{\infty}\left(0, T ; H_{0}^{1}(\Omega)\right) \cap L^{2}\left(0, T ; H_{0}^{1}(\Omega) \cap H^{2}(\Omega)\right) .
$$

\section{Limit of the approximate solutions}

From the estimates $(2.4),(2.13)$ and $(2.22)$, we can take the limit of the nonlinear system (2.1). In fact, there exists a subsequence of $\left(u_{m}\right)_{m \in N}$, which we denote as the original sequence, such that

$$
\begin{array}{lll}
u_{m}^{\prime} \longrightarrow u^{\prime} & \text { weak } & \text { in } L^{2}\left(0, T ; H_{0}^{1}(\Omega)\right), \\
u_{m} \longrightarrow u & \text { weak } & \text { in } L^{2}\left(0, T ; H_{0}^{1}(\Omega) \cap H^{2}(\Omega)\right) .
\end{array}
$$

Thus, by compact injection of $H_{0}^{1}(\Omega \times(0, T))$ into $L^{2}(\Omega \times(0, T))$ it follows by compactness arguments of Aubin-Lions [7], we can extract a subsequence of $\left(u_{m}\right)_{m \in N}$, still represented by $\left(u_{m}\right)_{m \in N}$ such that

$$
\begin{array}{ll}
u_{m} \longrightarrow u & \text { strong } \quad \text { in } L^{2}\left(0, T ; H_{0}^{1}(\Omega)\right), \quad u_{m} \longrightarrow u \quad \text { a.e. in } Q, \\
\nabla u_{m} \longrightarrow \nabla u & \text { strong } \quad \text { in } L^{2}(Q), \quad \nabla u_{m} \longrightarrow \nabla u \quad \text { a.e. in } Q .
\end{array}
$$

Let us analyze the nonlinear terms from the approximate system (2.1). From the first term, we know that

$$
\int_{\Omega}\left|a\left(u_{m}\right) \nabla u_{m}\right|^{2} \leq a_{1} \int_{\Omega}\left|\nabla u_{m}\right|^{2} \leq a_{1} C,
$$

and since that $u_{m} \rightarrow u$ a.e. in $Q$ and that $a(x,$.$) is continuous, we get$ $a\left(u_{m}\right) \longrightarrow a(u)$ and $\nabla u_{m} \longrightarrow \nabla u$ a.e. in $Q$. Hence, we also have

$$
\left|a\left(u_{m}\right) \nabla u_{m}\right|^{2} \longrightarrow|a(u) \nabla u|^{2} \quad \text { a.e. in } Q \text {. }
$$

From (2.25) and (2.26), and Lions Lemma, we obtain

$$
a\left(u_{m}\right) \nabla u_{m} \longrightarrow a(u) \nabla u \quad \text { weak in } L^{2}(Q) .
$$


From the second term, we know that

$$
\begin{aligned}
& \left.\left.\int_{0}^{T} \int_{\Omega}\left|b\left(u_{m}\right)\right| \nabla u_{m}\right|^{2}\right|^{2} \leq b_{1}^{2} \int_{0}^{T}\left|\nabla u_{m}\right|_{L^{\infty}(\Omega)}^{2} \int_{\Omega}\left|\nabla u_{m}\right|^{2} \\
& \leq b_{1}^{2} C_{0} \int_{0}^{T}\left\|u_{m}\right\|^{2}\left|\nabla u_{m}\right|_{H^{1}}^{2} \leq b_{1}^{2} C_{0}\left\|u_{m}\right\|^{2} \int_{0}^{T}\left|\Delta u_{m}\right|^{2} \leq C,
\end{aligned}
$$

where $C_{0}$ has been defined in (2.7).

By the same argument that leads to (2.26), we get

$$
\left.\left.\left.\left.\left|b\left(u_{m}\right)\right| \nabla u_{m}\right|^{2}\right|^{2} \longrightarrow|b(u)| \nabla u\right|^{2}\right|^{2} \quad \text { a.e. in } \quad \mathrm{Q}
$$

Hence, from (2.28) and (2.29), we obtain the convergence

$$
b\left(u_{m}\right)\left|\nabla u_{m}\right|^{2} \longrightarrow b(u)|\nabla u|^{2} \quad \text { weak in } L^{2}(Q) .
$$

Taking into account $(2.23),(2.27)$ and (2.30) into $(2.1)_{1}$, there exists a function $u=$ $u(x, t)$ defined over $\Omega \times[0, T$ [ with value in $\mathbb{R}$ satisfying (1.6). Moreover, from the convergence results obtained, we have that $u_{m}(0)=u_{0 m} \rightarrow u_{0}$ in $H_{0}^{1}(\Omega) \cap H^{2}(\Omega)$, and the initial condition is well defined.

Hence, we conclude that equation (1.6) holds in the sense of $L^{2}\left(0, T ; L^{2}(\Omega)\right)$.

\section{Uniqueness}

Let $w(x, t)=u(x, t)-v(x, t)$, where $u(x, t)$ and $v(x, t)$ are solutions of Problem (1.6). Then we have

$$
\left\{\begin{array}{l}
w^{\prime}-\operatorname{div}(a(u) \nabla w)-\operatorname{div}(a(u)-a(v)) \nabla v \\
\quad+b(u)\left(|\nabla u|^{2}-|\nabla v|^{2}\right)+(b(u)-b(v))|\nabla v|^{2}=0 \quad \text { in } Q \\
w=0 \quad \text { on } \Sigma, \quad w(x, 0)=0 \quad \text { in } \Omega .
\end{array}\right.
$$

Multiplying by $w(t)$, integrating over $\Omega$, we obtain

$$
\begin{aligned}
& \frac{1}{2} \frac{d}{d t}|w|^{2}+\int_{\Omega} a(u)|\nabla w|^{2} \leq \int_{\Omega}\left|\frac{d a}{d u}(\widehat{u})\right||w||\nabla v||\nabla w| \\
& +\int_{\Omega}|b(u)|(|\nabla u|+|\nabla v|)|\nabla w||w|+\left.\int_{\Omega}|\nabla v|^{2}|| \frac{\partial b}{\partial u}(\bar{u})|| w\right|^{2} \\
& \leq M \int_{\Omega}|\nabla w||w||\nabla v|+C_{0} \int_{\Omega}(|\nabla u|+|\nabla v|)|\nabla w||w| \\
& +M \int_{\Omega}|\nabla v|^{2}|w|^{2} \leq M|\nabla v|_{L^{\infty}(\Omega)}\|w\||w|+C_{0}\left(|\nabla u|_{L^{\infty}(\Omega)}+|\nabla v|_{L^{\infty}(\Omega)}\right)\|w\||w| \\
& +M|\nabla v|_{L^{\infty}(\Omega)}^{2}|w|^{2} \leq \frac{a_{0}}{2}\|w\|^{2}+\bar{C}\left(\left.\Delta u\right|^{2}+|\Delta v|^{2}\right)|w|^{2},
\end{aligned}
$$

where we have used 
(a) The generalized mean-value theorem, i.e.,

$$
|a(u)-a(v)|=\left|\frac{d a}{d u}(\widehat{u})(u-v)\right| \leq\left|\frac{d a}{d u}(\widehat{u})\right||w|, \quad u \leq \widehat{u} \leq v,
$$

(b) $\left|b(u)\left(|\nabla u|^{2}-|\nabla v|^{2}\right)\right| \leq|b(u)||\nabla w|(|\nabla u|+|\nabla v|)$,

(c) $|\nabla v|_{L^{\infty}(\Omega)} \leq\|\nabla v\|_{H^{1}(\Omega)} \leq\|v\|_{H^{2}(\Omega)} \leq|\Delta v|_{L^{2}(\Omega)}$.

The last inequality is valid only for the one-dimensional case.

Integrating (2.32) from 0 to $t$, we obtain

$$
\frac{1}{2}|w|^{2}+\frac{a_{0}}{2} \int_{0}^{t}\|w\|^{2} \leq \frac{1}{2}|w(0)|^{2}+\bar{C} \int_{0}^{t}\left(|\Delta u|^{2}+|\Delta v|^{2}\right)|w|^{2}
$$

where $\bar{C}$ denotes a different positive constant. Since $w(0)=u_{0}-v_{0}=0$, using the

Gronwall's inequality, we obtain $|w|^{2}+\int_{0}^{t}\|w\|^{2}=0$, which implies the uniqueness, $w(x, t)=u(x, t)-v(x, t)=0$ and the theorem is proved.

\section{Asymptotic Behavior}

In the following we shall prove that the solution $u(x, t)$ of Problem (1.6) decays exponentially when time $t \rightarrow \infty$, using the same procedure developed in Lions [7] and Prodi [10].

Theorem 3.1. Let $u(x, t)$ be the solution of Problem (1.6). Then there exist positive constants $\widehat{s}_{0}$ and $C=C\left\{\left\|u_{0}\right\|,\left|\Delta u_{0}\right|\right\}$ such that

$$
\|u\|^{2}+\left|u^{\prime}\right|^{2} \leq C \exp ^{-\widehat{s}_{0} t}
$$

Proof. To prove the theorem, complementary estimates are needed.

Estimate I': Consider the approximate system (2.1). Using the same argument as Estimate I, i.e, taking $v=u_{m}(t)$, we have

$$
\frac{d}{d t}\left|u_{m}\right|^{2}+\int_{\Omega} a\left(u_{m}\right)\left|\nabla u_{m}\right|^{2}+\int_{\Omega} b\left(u_{m}\right) u_{m}\left|\nabla u_{m}\right|^{2}=0
$$

Integrating (3.3) from $(0, T)$, we obtain

$$
\frac{1}{2}\left|u_{m}\right|^{2}+a_{0} \int_{0}^{T}\left\|u_{m}\right\|^{2}+\int_{0}^{T} \int_{\Omega} b\left(u_{m}\right) u_{m}\left|\nabla u_{m}\right|^{2}<\frac{1}{2}\left|u_{0}\right|^{2} .
$$

From H1 hypothesis, (3.2) and (3.3) we conclude

$$
a_{0}\left\|u_{m}\right\|^{2} \leq 2\left|u_{m}^{\prime}\right|\left|u_{m}\right| \leq 2\left|u_{m}^{\prime}\right|\left|u_{0}\right| .
$$


Estimate II': Taking derivative of the system (2.1) with respect to $t$ and taking $v=u_{m}^{\prime}$, we obtain

$$
\begin{aligned}
& \frac{d}{d t}\left|u_{m}^{\prime}\right|^{2}+a_{0}\left\|u_{m}^{\prime}\right\|^{2} \leq C \int_{\Omega}\left|u_{m}^{\prime}\right|^{2}\left|\nabla u_{m}\right|+\int_{\Omega}\left|u_{m}^{\prime} \| \nabla u_{m}\right|^{2} \\
& +C \int_{\Omega}\left|\nabla u_{m}\right|\left|\nabla u_{m}^{\prime} \| u_{m}^{\prime}\right| \leq C_{1}\left(\left\|u_{m}^{\prime}\right\|^{2}\left\|u_{m}\right\|+\left\|u_{m}^{\prime}\right\|^{2}\left\|u_{m}\right\|^{2}\right) .
\end{aligned}
$$

Hence,

$$
\frac{d}{d t}\left|u_{m}^{\prime}\right|^{2}+\frac{a_{0}}{2}\left\|u_{m}^{\prime}\right\|^{2}+\left\|u_{m}^{\prime}\right\|^{2}\left(\frac{a_{0}}{2}-C_{1}\left\|u_{m}\right\|-C_{1}\left\|u_{m}\right\|^{2}\right) \leq 0 .
$$

Using (3.4) then we can write the inequality (3.6) in the form,

$$
\frac{d}{d t}\left|u_{m}^{\prime}\right|^{2}+\frac{a_{0}}{2}\left\|u_{m}^{\prime}\right\|^{2}+\left\|u_{m}^{\prime}\right\|^{2}\left(\frac{a_{0}}{2}-C_{1}\left(\frac{2\left|u_{0}\right|}{a_{0}}\right)^{1 / 2}\left|u_{m}^{\prime}\right|^{1 / 2}-C_{1} \frac{2\left|u_{0}\right|}{a_{0}}\left|u_{m}^{\prime}\right|\right) \leq 0
$$

Let $v=u_{m}^{\prime}(0)$ in (2.1). Then, $\left.\left|u_{m}^{\prime}(0)\right|^{2} \leq\left.\left(C\left\|u_{0}\right\|+C_{2}\left|\Delta u_{0}\right|+\mid \Delta u_{0}\right)\right|^{2}\right)\left|u_{m}^{\prime}(0)\right|$ and we have

$$
\left.\left|u_{m}^{\prime}(0)\right|^{2} \leq\left(\left.C\left(\left\|u_{0}\right\|+\left|\Delta u_{0}\right|+\mid \Delta u_{0}\right)\right|^{2}+\left|\Delta u_{0}\right|^{3}\right)\right)^{2} .
$$

We define the operator

$$
\begin{aligned}
J\left(u_{0}\right)= & \left(\frac{2\left|u_{0}\right|}{a_{0}}\right)^{1 / 2}\left(C\left(\left\|u_{0}\right\|+\left|\Delta u_{0}\right|+\left|\Delta u_{0}\right|^{2}+\left|\Delta u_{0}\right|^{3}\right)\right)^{1 / 2} \\
& \frac{2\left|u_{0}\right|}{a_{0}} C\left(\left\|u_{0}\right\|+\left|\Delta u_{0}\right|+\left|\Delta u_{0}\right|^{2}+\left|\Delta u_{0}\right|^{3}\right) .
\end{aligned}
$$

Then we can show that

$$
\left(\frac{2\left|u_{0}\right|}{a_{0}}\right)^{1 / 2}\left|u_{m}^{\prime}(0)\right|^{1 / 2}+\frac{2\left|u_{0}\right|}{a_{0}}\left|u_{m}^{\prime}(0)\right| \leq J\left(u_{0}\right) .
$$

Consider $C_{1}$ a positive constant and $u_{0}$ small enough, that is,

$$
C_{1} J\left(u_{0}\right)<\frac{a_{0}}{4} .
$$

Then the following inequality holds,

$$
C_{1}\left(\frac{2\left|u_{0}\right|}{a_{0}}\right)^{1 / 2}\left|u_{m}^{\prime}\right|^{1 / 2}+C_{1} \frac{2\left|u_{0}\right|}{a_{0}}\left|u_{m}^{\prime}\right|<\frac{a_{0}}{4} \quad \forall t \geq 0 .
$$

Indeed, we can proof it by contradiction. Suppose that there is a $t^{*}$ such that

$$
C_{1}\left(\frac{2\left|u_{0}\right|}{a_{0}}\right)^{1 / 2}\left|u_{m}^{\prime}\left(t^{*}\right)\right|^{1 / 2}+C_{1} \frac{2\left|u_{0}\right|}{a_{0}}\left|u_{m}^{\prime}\left(t^{*}\right)\right|=\frac{a_{0}}{4} .
$$


Integrating (3.7) from 0 to $t^{*}$, we obtain $\left|u^{\prime}\left(t^{*}\right)\right|^{2} \leq\left|u^{\prime}(0)\right|^{2}$. From (3.11) and (3.12), we conclude that

$$
\begin{aligned}
& C_{1}\left(\frac{2\left|u_{0}\right|}{a_{0}}\right)^{1 / 2}\left|u_{m}^{\prime}\left(t^{*}\right)\right|^{1 / 2}+C_{1} \frac{2\left|u_{0}\right|}{a_{0}}\left|u_{m}^{\prime}\left(t^{*}\right)\right| \\
& \quad \leq C_{1}\left(\frac{2\left|u_{0}\right|}{a_{0}}\right)^{1 / 2}\left|u_{m}^{\prime}(0)\right|^{1 / 2}+C_{1} \frac{2\left|u_{0}\right|}{a_{0}}\left|u_{m}^{\prime}(0)\right| \leq C_{1} J\left(u_{0}\right)<\frac{a_{0}}{4} .
\end{aligned}
$$

Therefore, we have a contradiction by (3.13).

From (3.7), (3.12) and using the Poincaré inequality, we obtain

$$
\frac{d}{d t}\left|u_{m}^{\prime}\right|^{2}+s_{0}\left|u_{m}^{\prime}\right|^{2} \leq 0, \text { where } s_{0}=\left(a_{0} c_{0}\right) / 2 \text { and } c_{0} \text { is a positive constant such }
$$

that $\|\cdot\|_{H_{0}^{1}(\Omega)} \geq c_{0}|\cdot| L_{L^{2}(\Omega)}$. Consequently, we have $\frac{d}{d t}\left\{\exp ^{s_{0} t}\left|u_{m}^{\prime}\right|^{2}\right\} \leq 0$ and hence

$$
\begin{aligned}
\left|u_{m}^{\prime}\right|^{2} \leq\left|u_{m}^{\prime}(0)\right|^{2} \exp ^{-s_{0} t} & \leq C\left(\left\|u_{0}\right\|+\left|\Delta u_{0}\right|+\left|\Delta u_{0}\right|^{2}\right)^{2} \exp ^{-s_{0} t} \\
& \leq \widetilde{C}\left(\left\|u_{0}\right\|,\left|\Delta u_{0}\right|\right) \exp ^{-s_{0} t}
\end{aligned}
$$

We also have from (3.4) that

$$
\left\|u_{m}\right\|^{2} \leq \frac{2}{a_{0}}\left|u_{0}\right|\left|u_{m}^{\prime}\right| \leq \frac{2}{a_{0}} C\left(\left\|u_{0}\right\|,\left|\Delta u_{0}\right|\right)\left|u_{0}\right| \exp ^{-s_{0} t / 2} .
$$

Defining $\widehat{s}_{0}=s_{0} / 2$ then the result follows from $(3.15),(3.16)$ inequality and of the Banach-Steinhaus theorem.

Resumo A equação diferencial parcial não linear,

$$
u^{\prime}-\operatorname{div}(a(u) \nabla u)+b(u)|\nabla u|^{2}=0
$$

representa o problema de condução do calor em que os parâmetros, densidade de massa, calor específico e condutividade térmica são fortemente dependentes da temperatura. Nesse trabalho, provamos a existência, unicidade e o comportamento assintótico para o problema de valor inicial com apropriadas hipóteses sobre os parâmetros do material.

\section{References}

[1] N. Alaa and M. Iguername, Weak periodic solution of some quasilinear parabolic equations with data measures, Journal of Inequalities in Pure and Applied Mathematics, 3 (2002), 1-14.

[2] H. Amann, Periodic solutions of semilinear parabolic equations, Nonlinear Analysis, (1978), 1-29.

[3] L. Boccardo, F. Murat, and J.P. Puel, Existence results for some quasilinear parabolic equations, Nonlinear Analysis, 13 (1989), 373-392. 
[4] L. Boccardo and T. Gallouet, Nonlinear elliptic and parabolic equations involving measure data, Journal of Functional Analysis, 87 (1989), 149-168.

[5] M. Chipot and F.B. Weissler, Some blow up results for a nonlinear parabolic equation with a gradient term, SIAM J. Math. Anal., 20 (1989), 886-907.

[6] E. Feireisl, H. Petzeltová, and F. Simondon, Admissible solutions for a class of nonlinear parabolic problems with non-negative data, Proceedings of the Royal Society of Edinburgh, Section A - Mathematics, 131, No. 5 (2001), 857-883.

[7] J.L. Lions, "Quelques méthodes de résolution des problèmes aux limites non linéares", First edition, Dunod-Gauthier Villars, Paris, 1969.

[8] I-S. Liu, "Continuum Mechanics", Spring-Verlag, Berlin-Heidelberg, 2002.

[9] M. Nakao, On boundedness periodicity and almost periodicity of solutions of some nonlinear parabolic equations, J. Differential Equations, 19 (1975), 371385 .

[10] G. Prodi, Un Teorema di unicita per le equazioni di Navier-Stokes, Annali di Mat., 48 (1959), 173-182. 